\title{
Involvement of Local Communities and Households in the Implementation of the "One Health" Initiative Through the East African Integrated Disease Surveillance Network (EAIDSNet)
}

\author{
Stanley S. SONOIYA* \\ Health, East African Community Secretariat, Arusha, United Republic of Tanzania
}

\section{Objective}

The main objectives of the Network are to promote cross-border integrated diseases prevention and control through "One Health" approaches and joint action focusing on innovative human, animal and ecosystem health interventions, to among others:1) enhance and strengthen cross-country and cross-institutional collaboration through regional coordination of activities and local community participation, 2) promote exchange and dissemination of appropriate information on Integrated Disease Surveillance (IDS) and other disease control activities, 3) harmonize integrated disease surveillance systems in the region, 4) strengthen capacity for implementing integrated disease surveillance and control activities, and 5) ensure continuous exchange of expertise and best practices for integrated disease surveillance and control of pandemics and epidemics of communicable and vector-borne diseases in the East African Community Partner States (Burundi, Kenya, Rwanda, Uganda and Tanzania).

\section{Introduction}

The East African Community (EAC) is the regional intergovernmental organization of the Republics of Burundi, Kenya, Rwanda, the United Republic of Tanzania, and the Republic of Uganda, with its headquarters in Arusha, Tanzania. The East African Community (www.eac.int) is implementing the "One Health" initiative through the "East African Integrated Disease Surveillance Network (EAIDSNet)" which is a regional collaborative effort of the national Ministries responsible for human and animal health as well as the national health research and academic institutions of the five (5) EAC Partner States.

\section{Description}

Methodology: The "East African Integrated Disease Surveillance Network (EAIDSNet)" is implementing the "Community-Based Early Warning Integrated Disease Surveillance and Response" for the prevention and control of various human and animal diseases, parasites and pests, including involvement of local communities inter-facing with wildlife under the "One Health" initiative in the cross-border areas.

Conclusion: Involvement of local communities and households through participatory approaches in "One Health Initiative" leads to sustainable "Community-Based Early Warning Integrated Disease Surveillance and Response" for the prevention and control of various human and animal diseases, parasites and pests

\section{Audience Engagement}

Through oral presentations real life situations and experiences in the implementation of the East African Regional Transboundary Community-Based Integrated One Health Disease Prevention and Control Initiative in the Kagera River Basin Ecosytem involving the Republic of Uganda, the Republic of Burundi, the Republic of Rwanda and the United Republic of Tanzania

\section{Keywords}

One Health; EAIDSNet; Communities; disease; surveillance

\section{Acknowledgments}

Local communities and Volunteer Community Health Workers in the Kagera River Basin Ecosystem in the East African Transboundary region of Rwanda, Burundi, Uganda and Tanzania

*Stanley S. SONOIYA

E-mail: stanleysonoiya@gmail.com 